\title{
Imaging features of Vertebral Aneurysmal Bone Cyst and the clinical value of interventional embolization
}

\author{
QIAN ZHAO ${ }^{*}$, HAO XU ${ }^{2 *}$, XULI MIN ${ }^{2}$, LIN YANG $^{2}$ and YONGJUN REN ${ }^{2}$ \\ ${ }^{1}$ Department of Interventional Radiology, People's Hospital of Nanbu Country, Nanbu, Nanchong, Sichuan 637300; \\ ${ }^{2}$ Department of Interventional Radiology, Affiliated Hospital of North Sichuan Medical College, \\ Sichuan Key Laboratory of Medical Imaging, North Sichuan Medical College, Nanchong, Sichuan 637000, P.R. China
}

Received February 27, 2020; Accepted July 7, 2020

DOI: $10.3892 /$ etm.2020.9099

\begin{abstract}
The current study aimed to investigate the imaging manifestations of vertebral aneurysmal bone cyst (ABC), and examine the clinical value of interventional embolization. Imaging examinations of 5 patients with pathologically confirmed spinal ABC were analysed and arterial angiography and embolization were performed prior to surgery. The cases in this cohort exhibited different degrees of intrusion into the vertebral arch and spinous and transverse processes. Lesions that protruded into the spinal canal resulted in compression of the thecal sac and spinal cord. Enlargement of arteries was detected by digital subtraction angiography and decreased significantly following embolization. Blood loss during surgery was also significantly reduced. Preoperative embolization of $\mathrm{ABC}$ reduces bleeding and allows for easy access to tissue, which is of great clinical value.
\end{abstract}

\section{Introduction}

The aneurysmal bone cyst (ABC) was first defined by Jaffe and Lichtenstein, in 1942, as an intra-osseous and osteolytic lesion (1). ABC is a tumorous lesion with unknown aetiology, influencing the metaphyseal regions of the vertebrae and other bones (2). ABC is usually benign but may be locally aggressive, damaging the affected bones over time (3). ABC also exhibits high vascularity and does not often spontaneous heal $(4,5)$. ABC commonly occurs in adolescents and children, effecting $>70 \%$ of patients under 20 years old worldwide (4). The name ABC refers to its aneurysmal growth in the bone,

Correspondence to: $\mathrm{Dr} \mathrm{Hao} \mathrm{Xu}$, Department of Interventional Radiology, Affiliated Hospital of North Sichuan Medical College, Sichuan Key Laboratory of Medical Imaging, North Sichuan Medical College, 63 Wenhua Road, Nanchong, Sichuan 637000, P.R. China

E-mail: xuhao_niu@163.com

*Contributed equally

Key words: spine, aneurysmal bone cyst, embolism where it exhibits a cystic bulge full of blood (4). Common locations for $\mathrm{ABC}$ are the metaphysis, vertebral body, and iliac and long tubular bones $(4,6)$.

ABC occurring in the spinal column presents difficulties during surgical operation (7) due to unclear field of vision, increased blood loss and the possibility of not fully removing the lesions. The treatment strategies for ABC are embolization, injection with a fibrosing agent, intralesional curettage and resection $(4,8)$. Rigorous imaging evaluations prior to surgery and arterial embolization can reduce intraoperative bleeding and improve therapeutic efficacy and treatment compliance (9). In the current study, it was demonstrated that preoperative embolization is effective and has practical clinical value for spinal ABC.

\section{Materials and methods}

Study participants. A total of 3 males and 2 females were enrolled in the current study. Inclusive criteria: Diagnosis was confirmed by imaging and pathology; no other treatment, including drug therapy, was performed before endovascular embolization. Exclusion criteria: Secondary aneurysmal bone cyst; non vertebral aneurysmal bone cyst. These patients were between the ages of 12 and 27 years old and had been diagnosed with ABC at the Affiliated Hospital of North Sichuan Medical College and People's Hospital of Nanbu County from January 2015 to April 2018. This study was approved by the Ethical Committee of the Affiliated Hospital of North Sichuan Medical College and People's Hospital of Nanbu County (approval no. 2018 ER(A)12-027). Written informed consent was obtained from adult patients and legal guardian of minor patients. In these cases, lesions were located in the L1 (n=2), L2 ( $=1)$, T8 $(n=1)$ and T12 $(n=1)$ vertebrae (Table I). The main symptoms were lumbar back pain and local swelling. Although the neurological symptoms in this cohort were not obvious, there were signs of neurological issues, including radiating pain in the upper limb and chest, little finger abduction and iliopsoas muscle tenderness.

Arteriography and embolization therapy. The arteriography and embolization therapy were conducted as previously reported $(10,11)$ with a few modifications. Angiography was performed by puncture of the right femoral artery under local anaesthesia. A pigtail catheter was used for descending 
aortography to identify the intercostal and/or lumbar arteries involved in the lesion's blood supply. Depending on the supplying artery, an appropriate catheter was used for selective angiography. The spinal artery may originate from the posterior intercostal artery, necessitating the dilution contrast medium during angiography to prevent spinal cord injury. The degree of embolization was judged by blood flow velocity during embolization. When the blood flow was visibly slowed, gelatine sponge particles stopped being inserted and coils were pushed into the trunk of the supplying artery. After embolization, surgery was performed to remove the lesion 24-48 h later.

Haematoxylin and eosin staining. The resected bone tissue was fixed with $10 \%$ neutral phosphate buffered formalin (NBF) at $25^{\circ} \mathrm{C}$. After 1-2 days of fixation, the tissue block was dehydrated with gradient ethanol solution, washed using xylene, soaked in paraffin $\left(60^{\circ} \mathrm{C}\right)$ for $2 \mathrm{~h}$, and then embedded into a wax block. A Leica RM 2235 (Leica Biosystems) slicer was used for slicing. The slice thickness was $0.3 \mu \mathrm{m}$. Haematoxylin and eosin [Kilton Biotechnology (Shanghai) Co., Ltd.] was used to stain the nuclei and cytoplasm for 7-9 and 2-5 min, respectively, both at room temperature. After staining, gradient ethanol dehydration, washing with xylene and neutral gum sealing piece were successively carried out. Finally, sections were observed under a microscope (EM-300; Philips Healthcare) with magnification, $\mathrm{x} 400$.

\section{Results}

ABC occurred in the extraspinal part of the vertebral body in all 5 cases, showing expansive changes with varying degrees of bone involvement. Destruction and compression of the pedicle and spinous process were present (Fig. 1). The spinal canal at the corresponding level of the lesion was narrowed and the thecal sac was compressed with invasion of the right psoas major (Fig. 2).

Angiography indicated that the vessels most frequently supplying the lesions were the intercostal and/or lumbar artery, which were significantly thickened and showed spiral and tortuous forms, leading to compression in an arc in some cases (Fig. 3A). The lesions showed abnormal mass-like staining. The blood vessels were increased in number and disordered, displaying multi-vessel tumour-like changes, and the surrounding soft tissue formed small vessels of different sizes (Fig. 3A). Contrast media was patchy and irregular and no manifestations of arteriovenous fistula were identified. Following embolization, the vessels and lesions were not stained by the contrast media, indicating an adequate reduction in perfusion (Fig. 3B). Laminectomy and posterior fixation were performed 1-2 days after embolization (Fig. 4) and blood loss during surgery was between 500-1,000 ml. Images of haematoxylin and eosin staining were consistent with $\mathrm{ABC}$, and no obvious inflammatory or pathological changes were observed in the samples (Fig. 5). The figures presented represent the imaging manifestations and endovascular treatment of all 5 patients.

\section{Discussion}

$\mathrm{ABC}$ may occur at any segment of the spine and has different imaging characteristics at different stages and locations.
Radiographic manifestations such as lytic bone destruction, thin layer ossification, bone surface roughness, bone ridge and bone separation can be detected by X-ray $(12,13)$. As the lesion progresses, vertebral segments, including the vertebral arch and transverse process are damaged and become thinner, compressed and displaced, which may easily lead to pathological fracture (14). Computed tomography (CT) aids in the assessment of pedicle and vertebral body integrity prior to surgery. The presence of a multilocular cyst with a liquid-liquid interface on $\mathrm{T}_{2} \mathrm{WI}$ is highly suggestive of $\operatorname{ABC}(6,15)$. Inside the tumour, multiple cysts with fluid matter are present, revealing a different signal intensity in $\mathrm{T}_{1} \mathrm{WI}$ and $\mathrm{T}_{2} \mathrm{WI}$. These liquid-liquid manifestations are due to the varying degrees of blood oxidation and breakdown products in the cyst fluid $(16,17)$.

Treatment for some ABCs is difficult, especially in the spine and sacrum. For large frontal lesions, local excision or scraping is not easy and disease tissue may remain after surgery. Simultaneously, massive intraoperative bleeding is a significant problem (18). Current surgical treatments are not satisfactory in the balance of trauma and therapeutic effect and the occurrence of relapse is high (19). Radiotherapy and hormone therapy are also ineffective treatment and can lead to recurrence $(6,20)$. In addition, radiotherapy carries other risks such as myelopathy and radiation-induced spinal deformity. Interventional embolization can affect the lesion's hemodynamic features, thus promoting the repair process $(21,22)$. Identification of the blood vessels responsible for the tumour's blood supply is critical for preoperative embolization. The purpose of angiography is to understand the extent of the lesion, the relationship between multiple supply arteries and to identify the relationship between the spinal cord feeding arteries and the arteries supplying the lesion (23).

The blood supply in the spinal ABC is complex and involves multiple blood vessels (13). In general, the lesion is supplied by the intercostal artery and lumbar artery at the same level as the vertebra, but adjacent arteries may also be involved. Therefore, preoperative abdominal aortography is of great significance in assessing the number supplying arteries. This technique can also determine whether there are vascular co-trunks in the vessels supplying the spinal cord, providing visual confirmation for vascular selection and embolization.

It is crucial to select the appropriate size of the materials used for embolization because of the potential of breaking off during the procedure, leading to ectopic embolization (24). Therefore, the embolization process should be hyper-selective, target the vessel furthest from the lesion to avoid the remaining small co-trunk vessels, and be performed slowly under fluoroscopy. When the blood flow velocity is visibly slowed, the main trunk of the supply artery is adequately embolized with appropriately-sized micro-coils. After embolization of the supply artery, the demarcation between the tumour-like tissue and the surrounding normal tissue becomes more clear, which is beneficial for reducing bleeding, separating the tissue during surgery and improving the therapeutic effect of the treatment. In the present study, surgical operations, including curettage and resection, were performed 1-2 days after interventional embolization and it 
Table I. Baseline information of study participants.

\begin{tabular}{|c|c|c|c|c|c|c|c|}
\hline Sex & Age (years) & Region & Symptoms & $\begin{array}{l}\text { Signs of physical } \\
\text { examination }\end{array}$ & $\begin{array}{l}\text { Hospital stay } \\
\text { duration (days) }\end{array}$ & Blood loss (ml) & Treatment outcome \\
\hline M & 12 & L1 & Back pain & $\begin{array}{l}\text { Radiating right upper } \\
\text { limb pain }\end{array}$ & 17 & 500 & Symptoms relieved \\
\hline M & 18 & $\mathrm{~T} 8$ & $\begin{array}{l}\text { Pain and } \\
\text { local swelling }\end{array}$ & Radiating chest pain & 18 & 700 & Symptoms relieved \\
\hline M & 8 & L1 & Local swelling & $\begin{array}{l}\text { Flexion of left pinkie } \\
\text { and inability to abduct }\end{array}$ & 20 & 600 & Symptoms relieved \\
\hline $\mathrm{F}$ & 27 & L2 & Pain & $\begin{array}{l}\text { Tenderness of } \\
\text { iliopsoas }\end{array}$ & 18 & 1000 & Symptoms relieved \\
\hline $\mathrm{F}$ & 8 & T12 & Pain & None & 13 & 700 & Symptoms relieved \\
\hline
\end{tabular}

M, Male; F, Female.
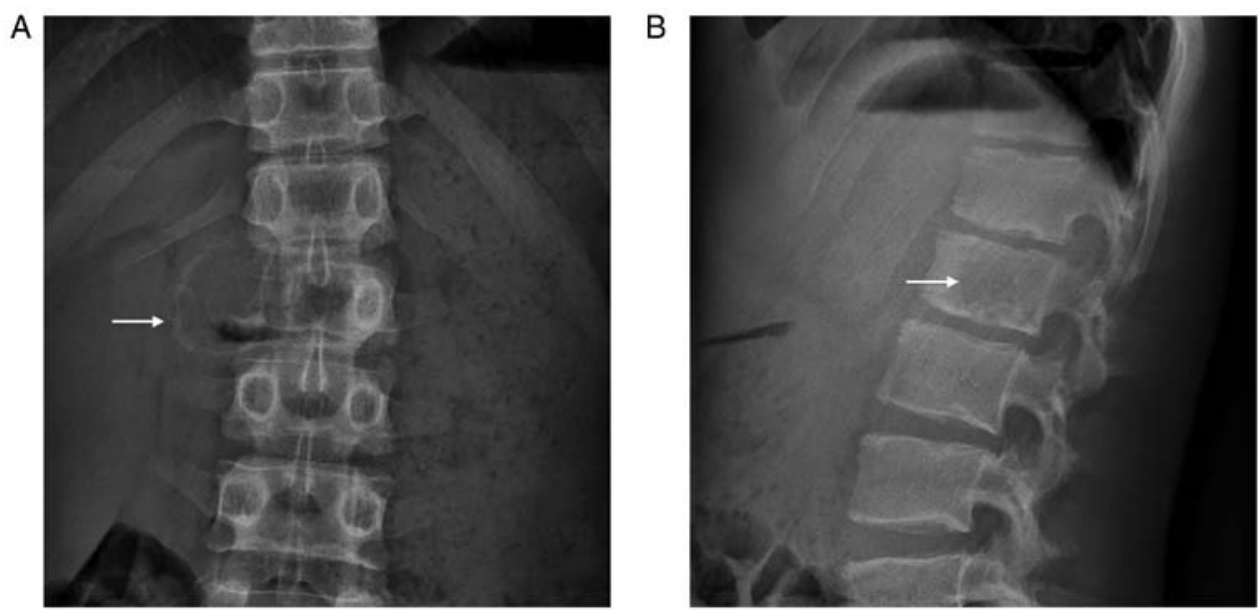

Figure 1. Preoperative X-ray showed lytic lesion involving the vertebral body, pedicle, lamina and spinous process at L1. (A) In the X-ray anteroposterior radiographs, L1 appendages were destroyed (white arrow). (B) Clear presence of sclerosis at the edge of the lesion (white arrow).
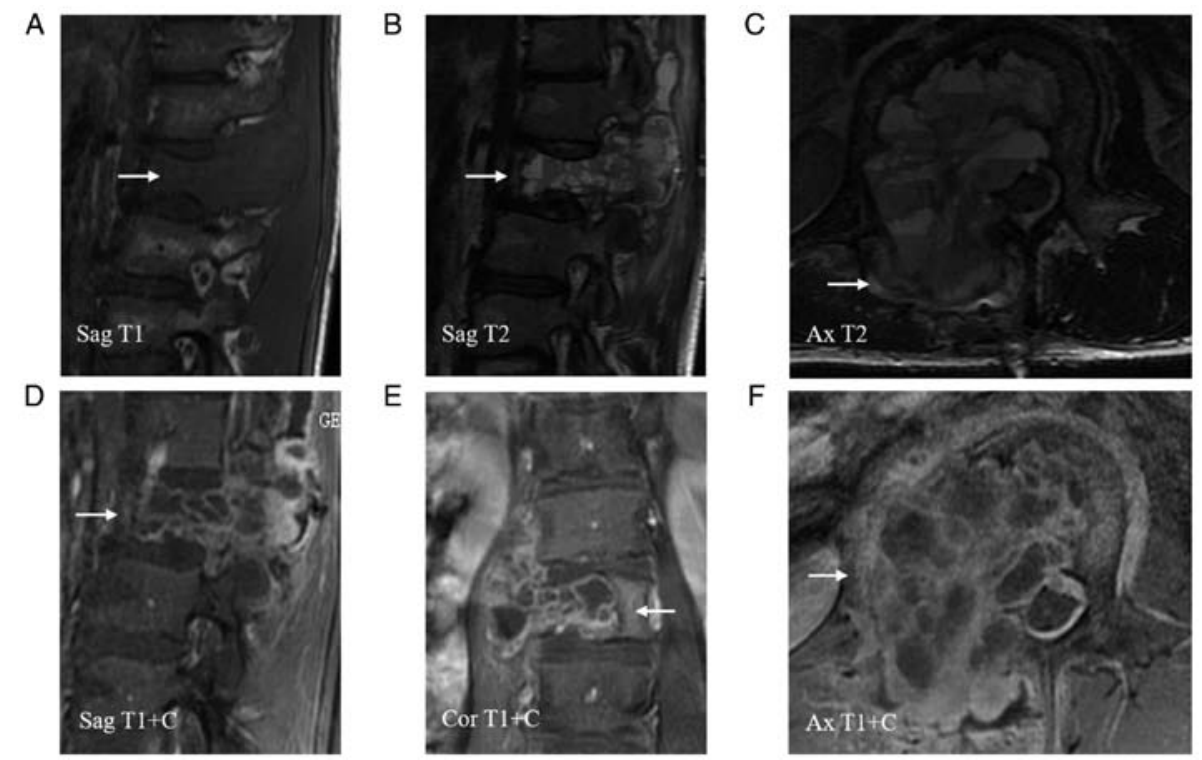

Figure 2. Preoperative MRI images of the same patient. (A) Sag $\mathrm{T}_{1} \mathrm{WI}$, the lesion showed the same low signal as muscle (white arrow). $\mathrm{T}_{2} \mathrm{WI}$ displayed a (B) heterogeneous and hyperintense lesion (white arrow; Sag image) and (C) fluid-fluid interfaces (white arrow) with thecal sac compression (Ax image). (D) Sag, (E) Cor and (F) Ax contrast magnetic resonance imaging on $\mathrm{T}_{1} \mathrm{WI}$ revealed a cystic section with heterogeneous enhancement of the solid part of the mass with prevertebral and paravertebral soft-tissue component (white arrow). Sag, sagittal; Cor, coronal; Ax, axial. 

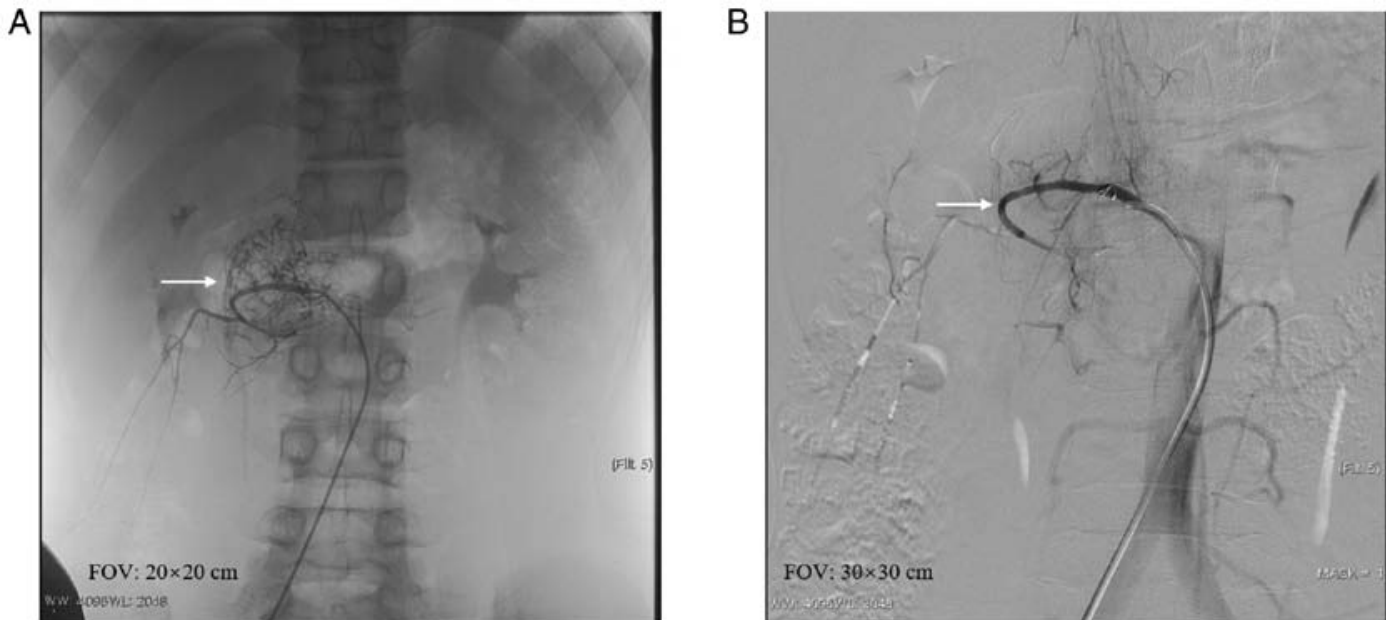

Figure 3. Images of arteriography and arterial embolization. (A) Angiography showed that the supply artery was enlarged, tortuous, and compressed in an arc shape and the lesion was visibly stained (white arrow). Gelatine particles and coils were injected into the lumbar artery for embolization. (B) Post-embolization angiograph displayed a significant reduction in staining (white arrow). FOV: Field of view.

A

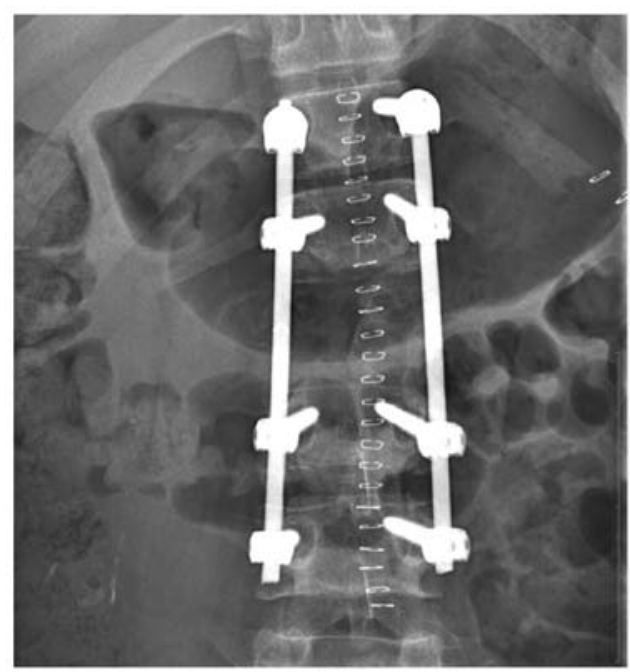

B

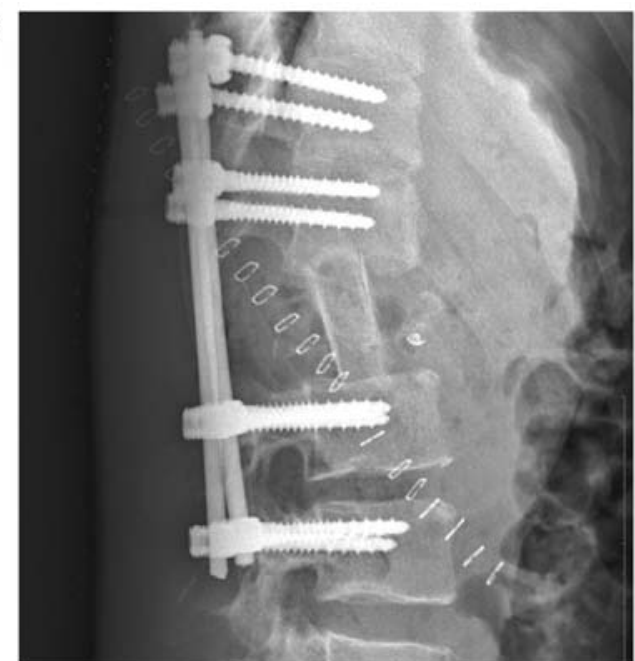

Figure 4. Postoperative X-ray images. Anteroposterior (A) and Lateral (B) radiograph showed complete tumour excision at L1 and the posterior lateral mass and pedicle screws.

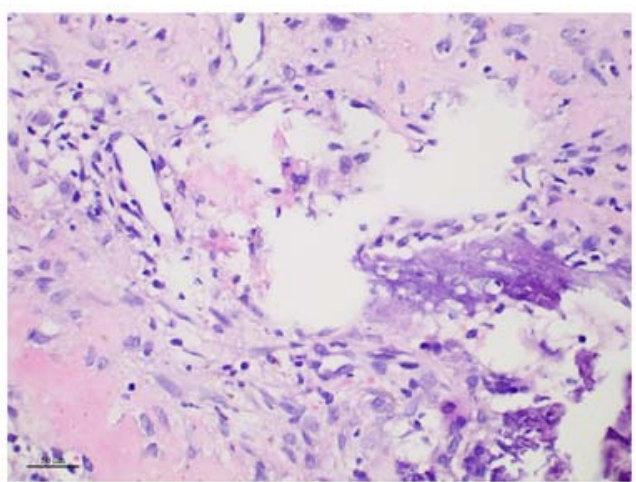

Figure 5. Representative histopathological haematoxylin \& eosin stain Magnification, x400.

was demonstrated that preoperative embolization was effective and is a valuable treatment method for this condition. Some studies have previously reported that zoledronic acid and other materials can be used to treat ABCs (25-27). In the cohort enrolled in the current study, preoperative embolization was only used as an adjuvant treatment in combination with other treatment methods, rather than a standalone treatment strategy.

The present study was a retrospective analysis, and no comparative analysis and case-control cohort were conducted. Additionally, the small sample size was also a limitation of the current study. In conclusion, interventional embolization can be used before surgery to optimise conditions for ABCs in challenging locations.

\section{Acknowledgements}

Not applicable.

\section{Funding}

No funding was received. 


\section{Availability of data and materials}

The datasets used and/or analysed during the current study are available from the corresponding author on reasonable request.

\section{Authors' contributions}

QZ and HX made substantial contributions to the conception and design of the study and wrote the original draft of the manuscript. XLM, LY and YJR were responsible for data acquisition and conducted data analysis and interpretation. HX revised the manuscript critically for important intellectual content. All authors read and approved the final version of the manuscript.

\section{Ethical approval and consent to participate}

This study was approved by the Ethical Committee of the Affiliated Hospital of North Sichuan Medical College and People's Hospital of Nanbu County (approval no. 2018 ER(A)12-027). Written informed consent was obtained from adult patients and legal guardian of minor patients.

\section{Patient consent for publication}

Not applicable.

\section{Competing interests}

The authors declare that they have no competing interests.

\section{References}

1. Jaffe HL: Aneurysmal bone cyst. Bull Hosp Joint Dis 11: 3-13, 1950.

2. Hetaimish BM and Alshaya OS: Pediatric aneurysmal bone cyst in the ischial region. Saudi Med J 37: 799-803, 2016.

3. Mankin KP, Bischoff RJ, Gelberman RH and Rosenberg AE: Aneurysmal bone cyst in involving the lunate. J Hand Surg Br 20: 12-15, 1995.

4. Cottalorda J, Kohler R, Sales de Gauzy J, Chotel F, Mazda K, Lefort G, Louahem D, Bourelle S and Dimeglio A: Epidemiology of aneurysmal bone cyst in children: A multicenter study and literature review. J Pediatr Orthop B 13: 389-394, 2004

5. Malghem J, Maldague B, Esselinckx W, Noel H, De Nayer P and Vincent A: Spontaneous healing of aneurysmal bone cysts. A report of three cases. J Bone Joint Surg Br 71: 645-650, 1989.

6. Parker J, Soltani S, Boissiere L, Obeid I, Gille O and Kieser DC: Spinal Aneurysmal bone cysts (ABCs): Optimal management. Orthop Res Rev 11: 159-166, 2019.

7. Mascard E, Gomez-Brouchet A and Lambot K: Bone cysts: Unicameral and aneurysmal bone cyst. Orthop Traumatol Surg Res 101 (Suppl 1): S119-S127, 2015.

8. Dormans JP, Hanna BG, Johnston DR and Khurana JS: Surgical treatment and recurrence rate of aneurysmal bone cysts in children. Clin Orthop Relat Res 421: 205-211, 2004.

9. Liu Y, Wang J, Lin L, Sang C, Lin Z, Pan Y and Fu X: Clinical study on complications of intracranial reptured aneurysm embolization by stent-assisted coil. Med Sci Monit 24: 8115-8124, 2018.
10. You Y, Choi SH, Choi DW, Heo JS, Han IW, Han S, Shin SW, Park KB, Park HS, Cho SK and Han SH: Long-term clinical outcomes after endovascular management of ruptured pseudoaneurysm in patients undergoing pancreaticoduodenectomy. Ann Surg Treat Res 96: 237-247, 2019.

11. Meyers PM, Shcumacher HC, Higashida RT, Derdeyn CP, Nesbit GM, Sacks D, Wechsler LR, Bederson JB, Lavine SD and Rasmussen P: Reporting standards for endovascular repair of saccular intracranial cerebral aneurysms. AJNR Am J Neuroradiol 31: E12-E24, 2010.

12. Hermann AL, Polivka M, Loit MP, Guichard JP and Bousson V: Aneurysmal bone cyst of the frontal bone-A radiologic-pathologic correlation. J Radiol Case Rep 12: 16-24, 2018.

13. Afnan J, Snuderl M and Small J: Intracranial, intradural aneurysmal bone cyst. Clin Imaging 39: 297-299, 2015.

14. Ghermandi R, Terzi S, Gasbarrini A and Boriani S: Denosumab: Non-surgical treatment option for selective arterial embolization resistant aneurysmal bone cyst of the spine and sacrum. Case report. Eur Rev Med Pharmacol Sci 20: 3692-3695, 2016.

15. Bazzocchi A, Spinnato P, Mercatelli D and Aparisi Gómez MP: Fluid-fluid levels in aneurysmal bone cysts. J Pediatr 204: 317, 2019.

16. Lopez LV, Roduriguez MG, Siegal GP and Wei S: Extraskeletal aneurysmal bone cyst: Report of a case and review of the literature. Pathol Res Pract 213: 1445-1449, 2017.

17. Baker KS, Gould ES, Patel HB and Hwang SJ: Soft tissue aneurysmal bone cyst: A rare case in a middle aged patient. J Radiol Case Rep 9: 26-35, 2015.

18. Mankin HJ, Homicek FJ, Ortiz-Cruz E, Villafuerte J and Gebhardt MC: Aneurysmal bone cyst: A review of 150 patients. J Clin Oncol 23: 6756-6762, 2015.

19. Steffner RJ, Liao C, Stacy G, Atanda A, Attar S, Avedian R and Peabody TD: Factors associated with recurrence of primary aneurysmal bone cysts: Is argon beam coagulation an effective adjuvant treatment? J Bone Joint Surg Am 93: e1221-e1229, 2011.

20. Patel RS, Dhamne CA, Gopinathan A, Kumar N and Kumar N: Denosumab: A potential treatment option for aneurysmal bone cyst of the atlas. Eur Spine J 27 (Suppl 3): S494-S500, 2018.

21. Rajput D, Tungaria A, Jaiswal A and Jain V: Aneurysmal bone cyst of clivus and $\mathrm{C} 1 \mathrm{C} 2$ : Case report and review of literature. Turk Neurosurg 22: 105-108, 2012.

22. Doss VT, Weaver J, Didier S and Arthur AS: Serial endovascular embolization as stand-alone treatment of a sacral aneurysmal bone cyst. J Neurosurg Spine 20: 234-238, 2014.

23. Patsalides A, Leng LZ, Kimball D, Marcus J, Knopman J, Laufer I, Bilsky M and Gobin YP: Preoperative catheter spinal angiography and embolization of cervical spinal tumors: Outcomes from a single Center. Interv Neuroradiol 22: 457-465, 2016.

24. Guo J, Yu J, Zhang Q and Song X: Clinical efficacy and safety of Utrine artery embolization (UAE) versus laparoscopic Cesarean scar pregnancy debridement surgery (LCSPDS) in treatment of Cesarean scar pregnancy. Med Sci Monit 24: 4659-4666, 2018.

25. Simm PJ, O'Sullivan M and Zacharin MR: Successful treatment of a sacral aneurysmal bone cyst with Zoledronic acid. J Pediatr Orthop 33: e61-e64, 2013.

26. Topouchian V, Mazda K, Hamze B, Laredo JD and Penneçot GF: Aneurysmal bone cysts in children: Complications of fibrosing agent injection. Radiology 232: 522-526, 2004.

27. Peraud A, Drake JM, Armstrong D, Hedden D, Babyn P and Wilson G: Fatal ethibloc embolization of vertebrobasilar system following percutaneous injection into aneurysmal bone cyst of the second cervical vertebra. AJNR Am J Neuroradiol 25: 1116-1120, 2004

(i) () () This work is licensed under a Creative Commons Attribution-NonCommercial-NoDerivatives 4.0 International (CC BY-NC-ND 4.0) License. 